\title{
Balanced Binary Search Trees Based Approach for Sparse Matrix Representation
}

\author{
Igor Balk ${ }^{1}$, Igor Pavlovsky ${ }^{2}$, Andrey Ushakov ${ }^{3}$, and Irina Landman ${ }^{3}$ \\ ${ }^{1}$ R3 Logic Inc, 80 Sherman Street, Camridge MA 02140, USA \\ 2 Terra Discount Club LLC, 110 Strathmore Rd 302, Brighton MA 02135 USA \\ ${ }^{3}$ Corning Ltd, 4 Birzhevaya Linia, 199034, St. Petersburg, Russia
}

\begin{abstract}
In this paper novel method of memory allocation for sparse matrixes is presented. Sparse matrixes are widely used in computational electrodynamics. This paper show that use of balanced binary search trees for memory allocation of sparse matrixes guarantees $\mathrm{O}(\ln (\mathrm{n}))$ access and insertion time. Comparison with traditional hash map memory allocation method was also made and presented in the paper.
\end{abstract}

\section{Introduction}

Many aspects of modern life require sophisticated computations to be performed. The majority of these computations and simulations are based on operations with sparse matrixes. A sparse matrix is a matrix with number of zero elements much larger than number of non-zero elements. These matrices are used in many computational disciplines such as image processing, computational electro and hydrodynamics and many others. Utilizing this characteristics, we can significantly reduce memory space required to store these matrixes and use special algorithms with this type of matrixes much faster than with dense matrixes. Good examples of such computations utilizing sparse matrices are image processing and electromagnetic simulation. Performance of mathematical libraries used for these simulations becomes critically important as complexity level growth. Direct algorithms will give $\mathrm{O}\left(\mathrm{n}^{3}\right)$, where $\mathrm{n}$ is the size of the system to be solved, increase in computational time and memory required, and it is challenging problem even for modern supercomputers. To solve this problem we developed fast numerical linear algebra library for sparse matrixes, which is based on data structures with $O(\ln (n))$ access time, where $n$ is dimension of the matrix.

Several data structures with $\mathrm{O}(\ln (\mathrm{n}))$ access time are known in graph theory. In our work we are utilizing different kinds of binary trees in order to accelerate performance of the library. The most natural assumption is to use red-black trees and AVL trees. These trees are well known in computer science theory and widely used for solution of network routing problem. We decided to use these trees to create a data structure in which sparse matrix will be stored. 


\section{Theory}

There are many publications available describing the theory of binary trees. These trees are popular since they allow the search of the element to be performed in $c^{*} \ln (n)$ operations, where $\mathrm{c}$ is some constant and $\mathrm{n}$ is the number of elements in the tree. The major and still unanswered question is how to reduce the constant $\mathrm{c}$. There are several techniques, which will give improvements in c value depending on the problem. Two most well known techniques are AVL trees and red-black trees. Use of this trees gives performance of a search and insertion operation in a tree close to optimal. $[1,2,3]$

A binary search tree (BST) is an AVL tree if the difference in height between the subtrees of each of its nodes is between -1 and +1 . Said another way, a BST is an AVL tree if it is an empty tree or if its subtrees are AVL trees and the difference in height between its left and right subtree is between -1 and +1 . In order to demonstrate performance we will mention some theorem [2]: an AVL tree with n nodes has height between $\log 2(\mathrm{n}+1)$ and $1.44 * \log 2(\mathrm{n}+2)-0.328$. An AVL tree with height $\mathrm{h}$ has between pow $(2,(\mathrm{~h}+.328) / 1.44)-2$ and pow $(2, \mathrm{~h})-1$ nodes.

For comparison, an optimally balanced BST with $n$ nodes has height ceil $(\log 2(n$ $+1)$ ). An optimally balanced BST with height $h$ has between pow $(2, h-1)$ and pow $(2, h)-1$ nodes. The average speed of a search in a binary tree depends on the tree's height, so the results above are quite encouraging: an AVL tree will never be more than about $50 \%$ taller than the corresponding optimally balanced tree. Thus, we have a guarantee of good performance even in the worst case, and optimal performance in the best case [2]. And for the red-black theorem, similar to the one we introduced for AVL trees [1,3], can be formulated: a red-black tree with n nodes has height at least $\log 2(\mathrm{n}+1)$ but no more than $2 * \log 2(\mathrm{n}+1)$. A red-black tree with height $\mathrm{h}$ has at least pow $(2, \mathrm{~h} / 2)-1$ nodes but no more than pow $(2, \mathrm{~h})-1$.

And for comparison, an optimally balanced BST with $n$ nodes has height ceil $(\log 2(n+1))$. An optimally balanced BST with height $h$ has between pow $(2, h-1)$ and pow $(2, h)-1$ nodes. Thus we can see that if we will use AVL and red-black trees to store data in sparse matrixes we will get performance close to optimal.

\section{Implementation}

Based of on the theory described above we have developed memory allocation algorithm for sparse matrixes. We used AVL trees to represent rows and colons of space matrixes. As it was shown above similar results could be obtained using redblack trees. Algorithm was implemented in ANSII C for maximum performance and portability. Important to mention, that described approach allows easy parallelization and further speed up on multiprocessor architecture.

\section{Computational Results}

We performed some preliminary tests comparing our new library with the data hash map memory allocation model, which is widely used in sparse matrix manipulation 
for example in such popular package as Matlab. Both memory models were implemented in ANSII C with same compiler optimization. We performed two tests to demonstrate performance of new memory model. The results of the tests are shown on Figures 1and 2.

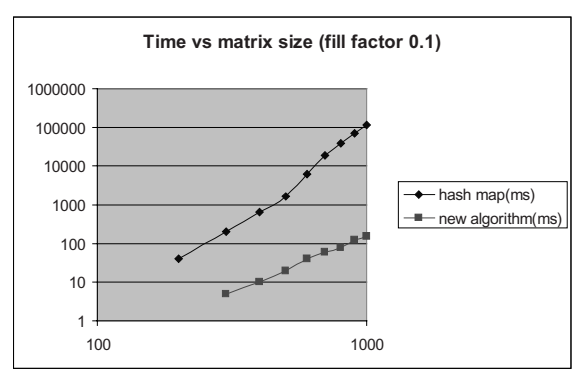

Fig. 1. Filling time vs. matrix size for fixed filling factor

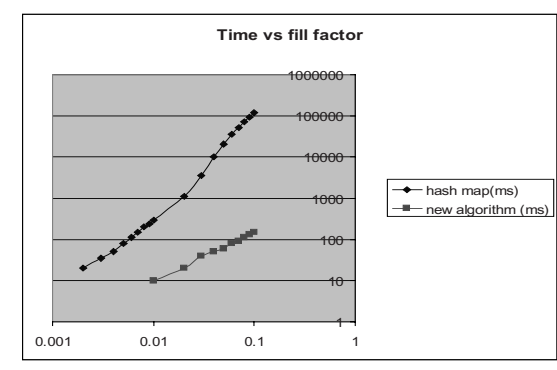

Fig. 2. Filling time vs. filling factor for fixed matrix size

On these figures dependence of the time of random filling, for the matrix of given size, from zero up to given filling factor is demonstrated. The Figure 1 demonstrates this dependence for the fixed filling factor $=0.1$ and matrix size varying from the $\mathrm{n}=10^{2}$ to the $\mathrm{n}=10^{3}$. On vertical axis filling time is shown and on horizontal - size of the matrix. As it's clear from the graph even with relatively small matrixes new algorithm of memory allocation gives order of magnitude saving in time. Figure 2 shows filling time (vertical axis) for the sparse matrix with fixed size and various filling factors (horizontal axis). The size of the matrix $\mathrm{n}$ is set to be $10^{3}$ and filling factor is varying from 0.002 to 0.1 .

Table 1. Speed of algorithm depending of filling factor for fixed matrix size ( $n=1000)$

\begin{tabular}{|r|r|l|}
\hline \multicolumn{1}{|l|}{ Fill factor } & \multicolumn{1}{|l|}{ Hash map (ms) } & New algorithm (ms) \\
\hline 0.003 & 35 & \\
\hline 0.004 & 50 & \\
\hline 0.005 & 80 & \\
\hline 0.006 & 110 & \\
\hline 0.007 & 150 & \\
\hline 0.008 & 200 & 10 \\
\hline 0.009 & 240 & 20 \\
\hline 0.01 & 291 & 40 \\
\hline 0.02 & 1122 & 50 \\
\hline 0.03 & 3646 & 60 \\
\hline 0.04 & 10344 & 80 \\
\hline 0.05 & 20900 & 90 \\
\hline 0.06 & 34840 & 110 \\
\hline 0.07 & 51143 & 130 \\
\hline 0.08 & 71342 & 150 \\
\hline 0.09 & 93955 & \\
\hline 0.1 & 117979 & \\
\hline
\end{tabular}


Table 2. Speed of algorithm depending of matrix size for fixed filling factor $(\mathrm{f}=0.1)$

\begin{tabular}{|r|r|r|}
\hline \multicolumn{1}{|l|}{ Matrix size } & Hash map (ms) & New algorithm (ms) \\
\hline 200 & 40 & \\
\hline 300 & 200 & 5 \\
\hline 400 & 630 & 10 \\
\hline 500 & 1623 & 20 \\
\hline 600 & 6329 & 40 \\
\hline 700 & 18307 & 60 \\
\hline 800 & 39156 & 80 \\
\hline 900 & 71403 & 120 \\
\hline 1000 & 117549 & 150 \\
\hline
\end{tabular}

\section{Conclusion}

We believe that the described library will provide engineers and researchers with a fast, reliable, and cost effective tool to satisfy the growing need for computational tools. A simple, intuitive interface, fast data access algorithms, and low cost will make this product a "must have" tool for people using numerical linear algebra in their work.

\section{References}

1. Cormen, C. H., C. E. Leiserson, and R. L. Rivest, Introduction to Algorithms. McGrawHill, 1990.

2. Knuth, D. E., The Art of Computer Programming, Volume 3: Sorting and Searching, 2nd ed. Addison-Wesley,

3. Sedgewick, R., Algorithms in C, Parts 1-4, 3rd ed. Addison-Wesley, 1998

4. Trefethen, Lloyd N.(Lloyd Nicholas)., David Bau, III, Numerical linear algebra SIAM, Philadelphia, 1997

5. Gene H. Golub, Charles F. Van Loan, Matrix Computations, 2 nd ed.The John Hopkins University Press, Baltimore, 1998

6. Igor Balk, Andrey Ushakov, Irina Landman "FNLAL: AVL-Tree based fast numerical linear algebra", Corning Technical Report, Corning, NY, 2001 